\title{
CORRESPONDENCE
}

\section{A CONJECTURE CHALLENGED AND WITHDRAWN}

Occasioned by Ingo Althöfer's article "Retrograde Analysis and Two Computerizable Definitions of the Quality of Chess Games" in the ICCA Journal, Vol. 12, No. 2, pp. 74-78, the Editor has received two letters, published in full below and amounting to Althöfer's withdrawing of one of his claims.

\section{A CONJECTURE CHALLENGED}

\author{
Peter Mysliwietz \\ University of Paderborn \\ Dept: of Mathematics and Computer Science \\ P.O. Box 1621 \\ 4790 Paderborn / West Germany
}

I would like to make a remark concerning Ingo Althöfers very interesting article in the ICCA Journal of June 1989.

In the introduction Althöfer described the problem to decide whether given any two chess positions $\mathrm{A}$ and $\mathrm{B}$ on an $\mathrm{n} \times \mathrm{n}$ board there is a legal chess game leading from $\mathrm{A}$ to $\mathrm{B}$. He conjectured that this question is undecidable. This is not true, simply because the number of chess positions on an $n \times n$ board is finite (dependent on $n$ ). Therefore a path-finding procedure for the reachability graph is sufficient to solve the problem.

\section{A CONJECTURE WITHDRAWN}

\author{
Ingo Althöfer \\ University of Bielefeld \\ Faculty of Mathematics \\ P.O. Box 8640 \\ 4800 Bielefeld / West Germany
}

Rainer Feldmann and Peter Mysliwietz have drawn my attention to a bad misser in my note "Retrograde Analysis and Two Computerizable Definitions of the Quality of Chess Games", in ICCA Journal, Vol. 12, No. 2, pp. 74-78. In the introduction I had conjectured that it might be impossible to decide whether there is a regular chess game from $\mathrm{A}$ to $\mathrm{B}$, where $\mathrm{A}$ and $\mathrm{B}$ are two positions on the generalized $\mathrm{n} \times \mathrm{n}$ board (instead of $8 \times 8$ ). However, for every fixed $\mathrm{n}$ there are only finitely many different chess positions on the $\mathbf{n} \mathbf{x} \mathbf{n}$ board. Hence it is, at least from the theoretical point of view, trivially feasible to decide whether there is a path from A to B in the finite directed graph which corresponds to the $\mathrm{n} \times \mathrm{n}$ chess.

The refuted conjecture of the note can be modified by looking at positions with finitely many figures on the infinite chess board (consisting of the whole plane). In this case the decidability status of the 'legal-game question' is a priori not clear as there are infinitely many positions.

I conjecture that also this case is decidable. Namely, it may be possible to prove something like the following statement:

"Let $\mathrm{A}$ and $\mathrm{B}$ be two positions on the infinite board such that in both all figures are standing on a subboard of size $\mathrm{n} \times \mathrm{n}$, and let $\mathrm{P}$ be an arbitrary regular game from $\mathrm{A}$ to $\mathrm{B}$. Then there exists a game $\mathrm{P}^{\prime}$ (maybe $\mathrm{P}=\mathrm{P}^{\prime}$ ) which also leads from $A$ to $B$, and in which all figures do not leave a finite subboard of size say $n^{2} \times n^{2}$ around the above-mentioned $\mathrm{n}$ x n subboard."

Such a statement would reduce the infinite board case to the finite one, which being denumerable is decidable. 